\title{
DARK MATTER IN GROUPS AND CLUSTERS OF GALAXIES
}

\author{
M. KALINKOV, I. KUNEVA AND I. VALTCHANOV \\ Institute of Astronomy, Bulgarian Academy of Sciences \\ 72 Tsarigradsko Chausse blvd, 1784 Sofia, Bulgaria \\ e-mail: markal@bgearn.bitnet, valtch@bgearn.bitnet
}

Samples of groups, clusters and superclusters of galaxies have been collected from existing catalogs. The group sample consisted of 70 systems, the list of clusters contains 50 systems, and there are five superclusters. Kinematical and dynamical characteristics are obtained - elongation (along the line of sight, RA and Dec), velocity statistics, total luminosity, harmonic radius, mean pair separation, crossing time, virial mass ... We have compared some of the observational features with dynamical models of groups, clusters and superclusters. Aarseth's (1985) code is used.

The most important results are

(i) Almost all investigated systems show substructures to a certain extent. The relative clumpiness is proportional to the hierarchy degree - it is smaller in groups and higher in superclusters.

(ii) It seems that dark matter is distributed predominantly in substructures. However it is very hard to distinguish this model from a continuous distribution over systems without any concentration.

A famous example of apparent subsuperclustering is the $\mathrm{CrB}$ supercluster. According to Kalinkov et al. $(1988,1989,1993)$ there are two superclusters in this region - CrB Near at $226 \mathrm{Mpc}(\mathrm{A} 2056, \mathrm{~A} 2065, \mathrm{~A} 2067, \mathrm{~A} 2079, \mathrm{~A} 2089$ and A2092) and CrB Distant at $360 \mathrm{Mpc}(\mathrm{A} 2049$, A2059, A2062, A2067B, A2069, A2083 and Z7412). There is a supervoid between both superclusters with $\mathrm{V}=1.2 \times 10^{6} \mathrm{Mpc}^{3}$.

We thank S. J. Aarseth for kindly providing us with his code NBODY2 and for numerous discussions and advices. This work was supported in part by the National Scientific Research Funds (contracts F107/1991 and F260/1992). 